\title{
Comprehensive Experimental Evaluation of a Systematic Approach for Cost Effective and Rapid Design of Condition Monitoring Systems using Taguchi's Method
}

\author{
A. Al-Habaibeh ${ }^{* a}$, F.Zorriassatine ${ }^{b}$, and N. Gindy ${ }^{b}$ \\ ${ }^{* a}$ Mechatronics Research Centre, Wolfson School of Mechanical and Manufacturing \\ Engineering, Loughborough University, LE11 3TU, UK \\ ${ }^{b}$ School of Mechanical, Materials, Manufacturing Engineering and Management, \\ University of Nottingham, NG7 2RD, UK
}

\begin{abstract}
This paper provides an extensive experimental and analytical evaluation of a previously presented approach to the systematic design of condition monitoring systems for machining operations [1]. The methodology termed Automated Sensor and Signal Processing Selection (ASPS), is based on Taguchi's orthogonal arrays in order to provide cost effective and speedy selection of sensors and signal processing methods that are ultimately used for monitoring process conditions. The evaluation using tool damage in end milling operations shows that ASPS methodology can successfully achieve its objectives without significantly affecting the system's capability for fault detection. The experiments investigate two new types of cutting tools each with three distinct conditions which are processed by four different and independent neural network paradigms - two supervised and two unsupervised. Thus, the results confirm the feasibility and efficiency of the proposed ASPS methodology and show that it can be applied to condition monitoring systems without the need for implementing pattern recognition tools during the design phase.
\end{abstract}

\footnotetext{
${ }^{*}$ Author to whom correspondence should be addressed.

Dr. Amin Al-Habaibeh, Mechatronics Research Centre, Wolfson School of Mechanical \&

Manufacturing Engineering, Loughborough, Leicestershire, LE11 3TU, UK, Tel: 01509226573 ,

e-mail: a.al-habaibeh@lboro.ac.uk
} 


\section{Keywords}

Condition monitoring system design, Taguchi's orthogonal arrays, Sensory fusion, Neural networks, End milling.

\section{Introduction}

The ultimate objective of automated condition monitoring systems for machining operations is to enhance the quality of manufactured products via detection of process and machine faults. During any machining operation a multitude of various signals are emitted from the machine tool and the process. Although these signals can provide useful inputs to a machine and process condition monitoring and diagnostic system, they usually include a significant amount of noise. Consequently, the 'raw' unprocessed signals are unsuitable for monitoring purposes. In order to extract useful information from machine condition monitoring data, several stages of signal processing and data analysis are normally needed. The ultimate goal of signal processing and data analysis is to search for the machining signals for abnormal patterns, i.e., Sensory Characteristic Features (SCFs), which can be related to physical phenomena or fault conditions.

The capability of a condition monitoring system relies on two basic elements: firstly, the number and type of sensors used and secondly, the associated signal processing and simplification methods utilised to extract important information from signals. Several successful industrial monitoring systems have been reported for monitoring continuous manufacturing processes such as those of paper mills [2], and chemical and metal production lines [3]. Other systems are designed for maintenance of machinery components such as compressors [4], bearings [5], gears [6], shafts [7], machine tool spindles [8], pumping systems [9] and fluid systems [10]. Nevertheless, a real industrial monitoring system design methodology for complex machining processes, such as end milling, is still needed.

The problem with machining processes is that they are complex and sophisticated, creating a unique design problem in every case. The main obstacles facing the designers of monitoring systems for machining operations are:

1. Selection of the number and type of sensors. 
2. Selection of the effective signal processing methods associated with the selected sensors.

3. Design of an effective fusion model (i.e., the combination of sensors and signal processing methods which give an improved performance).

4. Reduction in cost of machine and process monitoring systems without affecting the system's performance.

5. Automation of the design process.

A novel approach, known as Automated Sensor and Signal Processing Selection (ASPS), to solve these problems using an automated approach for the selection of sensor and signal processing methods for milling operations was recently reported [1]. The above technique resulted in reduced experimental work for rapid and cost effective design of condition monitoring systems for milling operations. The ASPS builds on the available knowledge in condition monitoring in order to advance the state of the art and provide a more generalised, simple and systematic approach to the design of condition monitoring systems for milling operations. Efficient design of condition monitoring systems is thus accomplished within a shorter development time, and more economically by minimising the number of sensors used. The main issue addressed in this paper is to provide an extensive evaluation and mathematical description of the ASPS methodology. In reference [1] only one type of cutting tool was examined using three conditions which were then processed by one neural network architecture, namely the Back-propagation network using supervised learning. The experiments reported here investigate an additional two types of cutting tools each with three distinct conditions which are in turn processed by four different and independent neural networks - two using supervised and the other two unsupervised learning. The paper then compares the results of the two papers and discusses the capability of the ASPS approach.

\section{A Brief Description of the ASPS Approach and Taguchi's Method}

\section{1 - The ASPS Approach}

The ASPS approach helps to design a condition monitoring system for a machine tool or a process using an automated simple procedure to detect the Sensory Characteristic Features (SCFs) which are most sensitive (i.e., dependent) to machine or process 
faults but less sensitive to other machining variables and parameters. The SCFs provide key information for classification or detection of machining faults. A SCF can be defined as an extracted sensory value from a sensory signal using a specific signal processing method, e.g., the average value of a force signal, or the standard deviations of an acceleration signal. The presented approach uses the "black box" concept where the monitoring system is designed based on the inputs and outputs of the process rather than its mechanics and the faults' mechanism. This "black box" approach gives the designer the ability to use the same approach of condition monitoring for other systems or processes. In this way, it is only required to relate some information in the machining signals (i.e., SCFs) to the identified faults or conditions. Figure (1) presents the main idea of "black box" concept and its applications in machining process. The "black box" should have the potential to move the design of condition monitoring problem from being a particular problem for a specific process to a more general problem that can be described in generic terms and the solution might be provided for different groups of processes that have specific criteria in common.

In order to reduce the experimental work needed to relate the sensitive sensory signals to process faults for many of the machining parameters, Taguchi's Orthogonal Arrays (OAs) were utilised in designing a short test, either on-line or off-line, to find out the most sensitive SCFs to the faults under investigation. The dependency values of Taguchi's OAs are taken as the measure of sensitivity of the SCFs to detect machining faults.

Based on a number of SCFs, a condition monitoring system can be designed by selecting the most sensitive group of SCFs, which show high dependency on the monitored faults. For example, a research study has shown that due to cutter wear there is a steady increase in the Root Mean Square (RMS) values of Acoustic Emission (AE) voltages of the work-piece [11]. It is therefore possible to use the increase in this sensory characteristic feature (average RMS level of AE voltage) as the basis for designing a condition monitoring system (i.e., the increase in the average RMS of AE signal indicates the development of cutter wear). 
Figure (2) shows a simplified flow diagram of the ASPS approach using Taguchi's method. The ASPS approach is performed by installing a variety of sensors on the machine tool. The obtained sensory signals are then processed using a wide range of signal processing methods. An automated procedure, using Taguchi's method, is then performed to choose a group of sensitive SCFs to build the required condition monitoring system. The least useful sensors and signal processing methods are eliminated from the designed condition monitoring system and only sensors and the associated signal processing methods which are found effective are kept in the monitoring system. Cost reduction stage is then performed for an attempt to eliminate the least utilised sensors in order to reduce the cost of the monitoring system while keeping the system's performance within its acceptable range. For further details please see reference [1].

\subsection{Taguchi's Orthogonal Arrays}

Taguchi's Orthogonal Arrays (OAs) are normally used to minimise the number of experiments required to characterise a process or optimise a process quality [12]. Taguchi's method uses less number of experiments than the full factorial to predict the influence of each experimental factor (i.e., independent variable) and calculate the dependency of the results on the experimental factors. The dependency values of Taguchi's method is basically the percentage contribution obtained from the analysis of variance. The dependency value of a result on a factor reflects the portion of the total variation observed in an experiment attributed to that factor. Based on Taguchi's method, the ASPS approach uses the SCFs obtained from the machining signals to calculate their dependencies (sensitivities) on the investigated machining faults. SCFs which show high dependency on the machining faults, rather than the machining parameters, are potential candidates for use in a monitoring system. The percentage contribution $(P)$ of a factor $(F)$ can be expressed as follows [13]:

$P_{F}=\frac{S S_{F}-V_{e} v_{F}}{S S_{T}} 100$

where

$S S_{F}=\sum_{i=1}^{K_{F}} \frac{F_{i}^{2}}{n_{F i}}-\frac{T^{2}}{M} \quad$ and $S S_{T}=\sum_{i=1}^{N} y_{i}^{2}-\frac{T^{2}}{M}$ 
$V_{e}$ is the variance due to the error and is given by

$$
V_{e}=\frac{S S_{T}-\sum_{F} S S_{F}}{M-1-\sum_{F} v_{F}}
$$

where:

$y$ : observation (i.e. sensory characteristic feature ( $\mathrm{SCF}$ ) value),

$T$ : sum of all observations (i.e. sum of all sensory characteristic feature (SCF) values),

$F$ : factor (i.e. cutter conditions, depth of cut, spindle speed, feed rate),

$K_{F}$ : number of levels for factor $F$ (i.e. $K_{F}=3$ in this research),

$M$ : total number of observations (i.e. $M=27$ in this research),

$v_{F}$ : is number of degrees of freedom associated with factor $\mathrm{F} ; v_{F}=K_{F}-1$,

$F_{i}$ : sum of observations under the $i$ th level of factor $\mathrm{F}$, and

$n_{F i}$ : number of observations $y$ under level $I$ of factor $F$.

In order for the approach to be useful, two main assumptions need to be tested, i.e.,

1. Partial number of runs using Taguchi's method is sufficient to design the monitoring system for full factorial runs; and

2. SCFs with high dependency values to a fault have high sensitivity to that fault.

\section{The Experimental Details}

\subsection{The Experimental Set-up}

The experimental work involves an end mill processing of aluminium parts using two additional different types of cutting tools for two independent experimental tests and design procedures. The cutters are $16 \mathrm{~mm}$ slot drill cutter and $16 \mathrm{~mm}$ four flutes screwed shank end mill cutter. The results of these two experiments are compared with those of the $16 \mathrm{~mm}$ four flutes end mill cutter described in reference [1]. The physical phenomena monitored in this research is teeth breakage of cutting tools. Every cutter is tested for three conditions: normal, one tooth broken, and two teeth broken.

Six accelerometers (Kistler 8704B500) are used to monitor the vibration of the spindle and the work-piece connected to two 4-channel-couplers (Kistler 5134). The 
acoustic emission signal is monitored using AE sensor (Kistler 8152A) which is connected to the AE-Piezotron coupler type (Kistler 5125). The latter produces the AE signal and the RMS of the AE signal. The force signals are monitored using 3component Dynamometer (Kistler 9257A). The force dynamometer is connected to a 3-channel charge Amplifier (Kistler 5001). The signals are monitored using a data acquisition card (National Instruments AT-MIO-64E-3). The experimental set-up is shown in Figure (3). Matlab software is used for the complete analysis of this work.

\subsection{The Experimental Methodology and Conditions}

The experimental program involves three values of depth of cut $(5,7$ and $9 \mathrm{~mm})$, spindle speed (2000, 2500 and $3000 \mathrm{rpm})$ and feed rate $(350,550$ and $750 \mathrm{~mm} / \mathrm{min})$ for every condition of the tested cutting tools. Figure (4) summarises the implemented experimental work.

A full factorial test of the parameters requires 81 runs for every cutting tool. However, the proposed application of Taguchi's method can reduce the number of runs to 27 runs using the $\mathrm{L}_{27}$ Table. For the three cutting tools, the experimental programme involved 27 runs based on OAs $\left(\mathrm{L}_{27}\right)$ and a further 81 full factorial runs. The experimental 27 test is used for the design process as well as training the neural networks. The full factorial test is used to test the capability of four neural networks to recognise cutter faults. Figure (5) presents the test program.

\section{Sensory Signals and Signal Processing Methods}

In addition to the eleven sensory signals shown in Figure (3), the design procedure includes another four sensory signals derived from the original signals. These signals are: the resultant acceleration of the spindle structure $(U R)$; the resultant acceleration of the work-piece $(L R)$; the resultant cutting force $(F R)$; and the net relative resultant acceleration between the spindle and the work-piece $(U L)$. The four signals are calculated based on the following equations:

$$
\begin{aligned}
& F R=\sqrt{(F x)^{2}+(F y)^{2}+(F z)^{2}} . \\
& U R=\sqrt{(X U)^{2}+(Y U)^{2}+(Z U)^{2}} .
\end{aligned}
$$


$L R=\sqrt{(X L)^{2}+(Y L)^{2}+(Z L)^{2}}$.

$U L=\sqrt{(X U-X L)^{2}+(Y U-Y L)^{2}+(Z U-Z L)^{2}}$.

where

$X U, Y U$ and $Z U$ : The spindle acceleration signals for the $\mathrm{x}, \mathrm{y}$ and $\mathrm{z}$ directions respectively.

$X L, Y L$ and $Z L$ : The work-piece acceleration signals for the $\mathrm{x}, \mathrm{y}$ and $\mathrm{z}$ directions respectively.

$F x, F y$ and $F z$ : Cutting force for the $\mathrm{x}, \mathrm{y}$ and $\mathrm{z}$ directions receptively.

In order to extract the SCFs, the fifteen signals are processed using several time and frequency domain signal processing methods to extract 23 SCFs from every sensory signal. The SCFs should be real numbers in order to use Taguchi's method to calculate the dependency values. The signal processing methods are chosen arbitrarily and any other type of signal processing methods might be used as long as they produce real numbers. The main objective is to produce simplified forms of the complex signal for later analysis. The signal processing methods used in the time domain are: the average $(\mu)$; standard deviations $(s t d)$; power [14]; kurtosis value $(K r)[15]$; and skew value [16]. In frequency domain two methods are used: the average values of different frequency ranges of Fast Fourier Transformation (FFT) [17]; and the standard deviations of Daubechie - D4 wavelet levels $[18,19]$. The description of the previous signal processing methods is given below:

1. average: $\mu=\frac{\sum_{i=1}^{N} x_{i}}{N} \quad$ where $x$ is the sensory signals

2. standard deviations: $\sigma=\sqrt{\frac{\sum_{i=1}^{N}\left(x_{i}-\mu\right)^{2}}{N-1}}$

3. power $\approx \frac{\sum_{i=1}^{N} x_{i}^{2}}{N}$

4. Kurtosis value $K r=\frac{1}{N} \sum_{i=1}^{N} \frac{\left(x_{i}-\mu\right)^{4}}{\sigma^{4}}$ 
5. $S k e w=\frac{1}{N} \frac{\sum_{i=1}^{N}\left(x_{i}-\mu\right)^{3}}{\sigma^{3}}$

\subsection{Fast Fourier Transformation Analysis}

The Fast Fourier Transformation (FFT) [17] algorithm is used to convert a digital signal $(x)$ with length $(N)$ from the time domain into a signal in the frequency domain $(X)$.

$X[h]=\sum_{i=0}^{N-1} x[i] W_{N}^{i h} \quad$ Where $\quad W_{N}=e^{-J 2 \pi / N}$.

for $h=0,1,2, \ldots, N-1$ and where $J=\sqrt{-1}$

In order to automate the selection process of the sensitive frequencies to the fault under investigation, the frequency spectrum vector is divided into seven ranges of frequencies. The average value of each range is then taken as a sensory feature for the system. The values of the frequency spectrum are normalised with respect to the amplitude. The width of every range and the number of ranges are selected so that the average of the ranges can represent the actual frequency spectrum. These features calculated as the average of the frequency spectrum values in specific range of frequencies. For example, in this particular case, FFT1 is the average value of the frequencies from $0.09 \mathrm{kHz}$ to $1.09 \mathrm{kHz}$. In general:

FFT $i\left(f r_{1} f r_{2}\right)=$ Mean value of the Fast Fourier Transformation between frequencies frl and fr2;

Where:

$i=\{1,2,3,4,5,6,7\}$

$f r_{1}=0.09+(1.01 \times(i-1)) \mathrm{kHz}$

$f r_{2}=1.09+(1.01 \times(i-1)) \mathrm{kHz}$

Every feature has a range of frequency width of $1 \mathrm{kHz}$. The number of ranges and the width of each range are, in general, dependent on the type of signal and its application. The narrower the range, however, the more accurate the analysis will be while requiring more calculations and longer time to accomplish.

\subsection{Wavelet Analysis}


In this work, the standard deviations of 11 wavelet levels are used as SCFs for the condition monitoring system. Wavelet analysis provides an alternative method of breaking a signal down into sub-signals or levels with different frequencies that carry the time information $[18,19]$. Wavelet analysis breaks up the signal into sub-signals where each one is generated by a shifted and scaled of several wavelet signals. For every level, the number of wavelet signals used to construct the signal equals $2^{i}$ where $i$ is the level number. The dilation equation is used to define the basic scaling function $\phi(x)$ from which the D4 discrete wavelet original signal is calculated according to the following equation:

$$
\Phi(x)=\sum_{i=0}^{3} c(i) \Phi(2 x-i)
$$

where $c(i)$ is the wavelet coefficient and $i$ is the index.

The Primary wavelet signal is calculated from the scaling function according to the equation shown below:

$$
\Psi(x)=\sum_{i=0}^{3}(-1)^{i} c(i+1) \Phi(2 x-i)
$$

The four coefficients for D4 wavelet are as follows:

$$
\begin{array}{ll}
c(0)=(1+\sqrt{3}) / 4 \quad ; & c(1)=(3+\sqrt{3}) / 4 \\
c(2)=(3-\sqrt{3}) / 4 \quad ; & c(3)=-(\sqrt{3}-1) / 4
\end{array}
$$

For discrete D4 wavelet transformation, the original function can be reconstructed from the following equation:

$$
f(x)=\sum_{l=-\infty}^{\infty} c_{\Phi, l} \Phi_{n}(x-l)+\sum_{\mathrm{i}=0}^{\infty} \sum_{h=-\infty}^{\infty} c_{h, l} \Psi\left(2^{h} x-l\right)
$$

The standard deviations $(\sigma)$ of the wavelet levels are used as sensory characteristic features for the condition monitoring system. In general, the eleven wavelet SCFs used is denoted as ( $s t d L i)$ where $s t d L i$ is the standard deviations of the $i$ th level of the D4 wavelet.

\section{The Experimental Results}




\subsection{The ASPS analysis of the experimental work.}

The calculated Sensory Feature Matrix (SFM) for this test, see reference [1], has dimensions of $(15 \times 23 \times 27)$ thus presenting 15 sensory signals, signal processing methods and 27 runs of is the L27 Orthogonal Array. For every feature located in the SFM matrix, the dependency on the cutter conditions is calculated and placed in the Association Matrix (ASM). Consequently, the ASM matrix for the cutter conditions has a size of $15 \times 23$, making a total of 345 SCFs. The dependency coefficients of the ASM are used as an indicator of the sensitivity of the features to cutter conditions. The $345 \mathrm{SCF}$ are divided into 17 different groups/systems where each system contains 20 features. The features are arranged in a descending order so that system number 1 contains the features of maximum dependencies while system number 17 contains the features of minimum dependency. The suggested number of 20 features in every system is based empirically on the author's previous experience with condition monitoring and neural networks. Normally, such a range of inputs provides good identification and relatively fast training time. However, other values might also be used depending on the application and the neural networks topology. The monitoring systems with each consisting of 20 SCFs, includes SCFs from different sensors using different signal processing methods.

\subsection{Neural Networks Classification}

As previously mentioned, four neural networks are used to verify whether that SCFs with higher dependency can indeed provide a greater sensitivity that should consequently result in better identification of abnormal patterns (see Figure 5). The neural networks are two supervised and two unsupervised neural networks implemented using Matlab. The neural network architectures included: back propagation $(\mathrm{BP})$; radial basis $(\mathrm{RB})$; Competitive Neural Network $(C N)$; and Learning Vector Quantisation $(L V Q)$, see Figure (6).

Since the 17 proposed systems have 20 SCFs each, the neural networks implemented here are designed to have 23 inputs, where the extra three inputs are for the normalised cutting conditions (i.e. feed rate, depth of cut and spindle speed). 
A normalising process is performed using equation (20) below so that every sensory characteristic feature will have a value between 0.1 and 0.9 thus making it possible to fuse and compare all the calculated sensory features relative to each others.

$$
\begin{aligned}
\hat{f}_{i j}= & 0.1+\frac{0.8}{\max -\min } \times\left(f_{i j}-\min \right) \\
& \text { where } \\
& \text { max: is the maximum value of the feature } f_{i j} . \\
& \text { min: is the minimum value of the feature } f_{i j} . \\
& \hat{f}_{i j}: \text { normalised values of the feature } f_{i j}
\end{aligned}
$$

The neural network parameters are chosen from experience in order to give a reasonable response, however, it is important to point out that neural networks are not optimised for this application since the objective here is to compare systems in order to select the most appropriate sensors and signal processing methods. The $\mathrm{L}_{27}$ runs are used to train the neural networks while the full factorial tests are used to test them, (i.e. using new 81 runs). Although the 81 new runs contain 54 runs which have different machining parameters, this should not pose a problem for the neural networks since the sensory characteristic features which show high dependency on the cutter conditions should show low dependency (sensitivity) to the other machining parameters. A total of 40 independent training and testing processes are performed for each tested system. The average classification errors of the BP, RB, CN and LVQ neural networks for the three cutting tools, end mill [1], slot drill, and screwed shank cutters are shown in Figure (7), Figure (8) and Figure (9) respectively. As shown in the previous figures, there is a clear trend that systems with high average dependency values produces less classification error (i.e. better identification). Moreover, for systems with dependency greater than $45 \%$, the results are more steady and have lower average variation relative to each others. Therefore, it can be concluded that the higher the average dependency of the system, the better and more stable the classification of the pattern recognition system. The computer calculation of the dependency values for each system only takes a fraction of a second. On the other hand, training and testing the neural networks to plot Figure (7), for example, has required about 250 hours calculations using a Pentium 266 computer with $120 \mathrm{MB}$ of memory and using Matlab software. 
The ASPS approach is found to be very useful in predicting the behaviour of condition monitoring systems without the need to use any iterative methods. The average classification errors of the four neural networks have proved that high dependency means better information for the neural networks, see Figure (5).

In comparison with other neural networks, the competitive neural network $(C N)$ is found to be divergent when applied to the slot drill cutter. It also displays higher errors when used with the screwed shank cutter. The general behaviour of neural networks, however, has shown the same results for the three cutters. The back propagation neural network is chosen for further analysis regarding the performance of the systems since it shows a stable and average performance relative to other neural networks.

\section{Sensors Utilisation and Cost Reduction}

In order to maintain the cost of the monitoring system as low as possible, the utilisation of sensors in the system should be kept as high as possible (i.e. getting as much information as possible from each sensor). The utilisation factor (SU) of a sensor is defined in equation (21) below.

$S U=\frac{v}{q x w} \times 100$

where:

$v: \quad$ number of sensory characteristic features used from the sensor

$q$ : total number of features in the system (20 is chosen in this case)

$w$ : maximum number of signals that can be produced by the sensor

The overall Average Sensor Utilisation factor for a system (SUA) is defined as the average value of the $S U$ of all the sensors used in a system. The $S U$ factor can be useful in reducing the cost of the system by eliminating the least utilised sensors in a monitoring system. The variable nominal cost of each system is calculated and compared in an attempt to optimise the performance of the system relative to its cost. The cost analysis is based on using the same equipment described in section 3.1. The cost values presented in this paper are only examples of the relative costs involved, which help to explain the methodology. These values are used for comparison 
purposes and should not be considered as real market values of the sensors. The cost analysis is done using the variable cost of the system, i.e. the cost of the sensors. Fixed costs such as the PC, data acquisition card, and the software cost should be added to the nominal variable cost in order to get the total cost of the system. In this research, the term "cost" refers to the nominal variable cost of the monitoring system as the objective is only to compare systems.

\subsection{Cost reduction for the monitoring system of the slot drill cutter.}

Figure (10) shows the 17 proposed systems for the slot drill cutting tool condition monitoring. Figure (11) presents the utilisation of (system 1) in order to reduce system's cost. The new optimised system is found to have a cost of $£ 809$ and an average dependency of $45.1 \%$. The new value is not significantly different from the original value of $47.54 \%$ for the first system. However, the new system is $£ 15,200$ cheaper than system number 1 . The performance of the optimised system relative to the first systems is also shown in Figure (11).

\subsection{Cost reduction for the monitoring system of the screwed shank cutter.}

Figure (12) shows the 17 proposed systems for the screwed shank cutting tool condition monitoring. It can bee seen that system 1 has the lowest cost and the lowest neural networks classification error. However, an optimisation of the cost is performed, see Figure (11), to reduce the cost by $£ 1952$ using the force dynamometer and removing all other sensors from the system. The performance of the optimised system with comparison to the average dependency value and cost is also shown in Figure (11).

\section{Conclusion}

Further experimental work and analysis to confirm the applicability of the ASPS approach was presented. Reference [1] used an end mill cutter to provide the scientific basis for the methodology. In this paper, two new sets of additional experimental work are performed using a slot drill and a screwed shank cutter, and four types of neural networks. 
For every test, the most suitable sensor is found to be different. As shown in Figure (11), some sensors are more useful than others in monitoring a specific type of cutter. For example, the force dynamometer is significant for monitoring the screwed shank cutter, but not for the other two cutters. The acoustic emission sensor is useful for the end and slot mills and not significant for the screwed shank cutter. As for the end mill cutter [1], the accelerometers mounted on the machine table have a higher utilisation and significance compared to those on the spindle housing. This observation can be explained by the fact that the SCFs that are sensitive to cutter faults are different for every cutter. Each cutter has its own specific nature and the generated faults produce different types of signals and frequencies. Therefore, every sensor extracts different information about the fault. The sensor which extracts more information is more likely to provide the sensitive SCFs for the monitoring system. The mechanism of the end milling process and the relationship between the generated faults and the produced signals are beyond the scope of this paper and can be investigated by future research.

The results confirm that only a partial number of the experimental tests are required in order to predict the machining condition for the full combinations of machining parameters and machining faults.

The approach is proved to benefit the design process by :

- Investigating the most appropriate sensors and signal processing method to detect machining faults,

- The reduction in cost of machine and process monitoring systems without affecting the system's performance using a suitable fusion model,

- Reducing the development time, and

- The automation of the design process. 


\section{References}

1. A. Al-Habaibeh, N.Gindy, "A new approach for systematic design of condition monitoring systems for milling processes", Journal of Materials Processing Technology, 107 , pp 243-251, 2000

2. A. Foster, "Ten Years of Condition Monitoring Changes and Causes", 5th International Conference on Profitable Condition Monitoring Fluids and Machinery Performance Monitoring, Mechanical Engineering Publications Limited, UK, pp 3-21, 3-4 December 1996.

3. A. Davies, "Handbook of Condition Monitoring -Techniques and Methodology", Chapman \& Hall, London, UK, 1998.

4. M. N. M. Badi, M. Carvell, and S. N. Engin, "Condition monitoring of a pair of industrial compressors", 5th International Conference on Profitable Condition Monitoring Fluids and Machinery Performance Monitoring, Mechanical Engineering Publications Limited, UK, pp 81-98, 3-4 December 1996.

5. R. B. Heng, M. J. Mohd Nor, "Condition monitoring Using Acoustic Signals", 5th International Conference on Profitable Condition Monitoring Fluids and Machinery Performance Monitoring, Mechanical Engineering Publications Limited, UK, pp-145-158, 3-4 December 1996.

6. J. C. Robinson, R. G. Canada, and K. R. Piety, "Vibration Monitoring on Slow Speed Machinery: New Methodologies Covering Machinery from 0.5 to 600 RPM", 5th International Conference on Profitable Condition Monitoring Fluids and Machinery Performance Monitoring, Mechanical Engineering Publications Limited, UK, pp 169-181, 3-4 December 1996.

7. D. U. Mba, R. H. Bannister, "Profitable Condition Monitoring of Low Speed Rotating Machinery Using Stress Waves", 5th International Conference on Profitable Condition Monitoring Fluids and Machinery Performance Monitoring, Mechanical Engineering Publications Limited, UK, pp 159-168, 3-4 December 1996.

8. H. Habermann and G. Liard, " An Active Magnetic Bearing System", Precision Engineering, Vol 6, No. 3,1984.

9. M. Carlos Talbott, “Diagnosis and Prognosis of Large Horsepower Electric Submersible Pumps", The $13^{\text {th }}$ International Congress on Condition Monitoring 
and Diagnostic Engineering Management (COMADEM 2000), MFPT Society, Houston, Texas, USA, December 3-8, 2000.

10. M. J. Day, "Condition Monitoring of Fluid Systems - The Complete Approach", 5th International Conference on Profitable Condition Monitoring Fluids and Machinery Performance Monitoring, Mechanical Engineering Publications Limited, UK, pp 183-206, 3-4 December 1996.

11. J. S. Kim, M.C. Kang, B. J. Yyu and Y. K. Ji, " Development of an on-line toollife monitoring system using acoustic emission signals in gear shaping, International Journal of machine tools and Manufacture Vol. 39, No. 11, pp 1761$1777,1999$.

12. A. Bendell, J. Disney and W. A. Pridmore, "Taguchi Methods, Applications in World Industry", IFS publications, UK, 1989.

13. P. J. Ross, "Taguchi Techniques for quality Engineering", McGraw-Hill, NewYork, 1988.

14. M. Etter, "Engineering Problem Solving with Matlab", Englewood Cliffs : Prentice Hall, p 147, 1993.

15. Z. Wang and D. A. Dornfeld, "In-Process Tool Wear Monitoring Using Neural Networks", Japan/USA Symposium on Flexible Automation, ASME, Vol.1, part 1, pp 263-270, 13-15 July 1992.

16. L. Blank, "Statistical Procedures for Engineering, Manufacturing, and Science", McGraw-Hill, Inc., 1980, PP 66-72

17. C. S. Burrus, J. H. McClellan, A. V. Oppenheim, T. W. Parks, R.W. Schafer, H. W. Schuessler, "Computer-based exercises for signal processing using MATLAB", Prentice Hall International, pp43-59, 1994.

18. D. E. Newland, "An Introduction to Random Vibrations, Spectral and Wavelet Analysis", Longman Scientific and Technical, 1993.

19. G. Strang and T. Nguyen, "Wavelets and Filter Banks", Wellesley -Cambridge Press, 1997. 


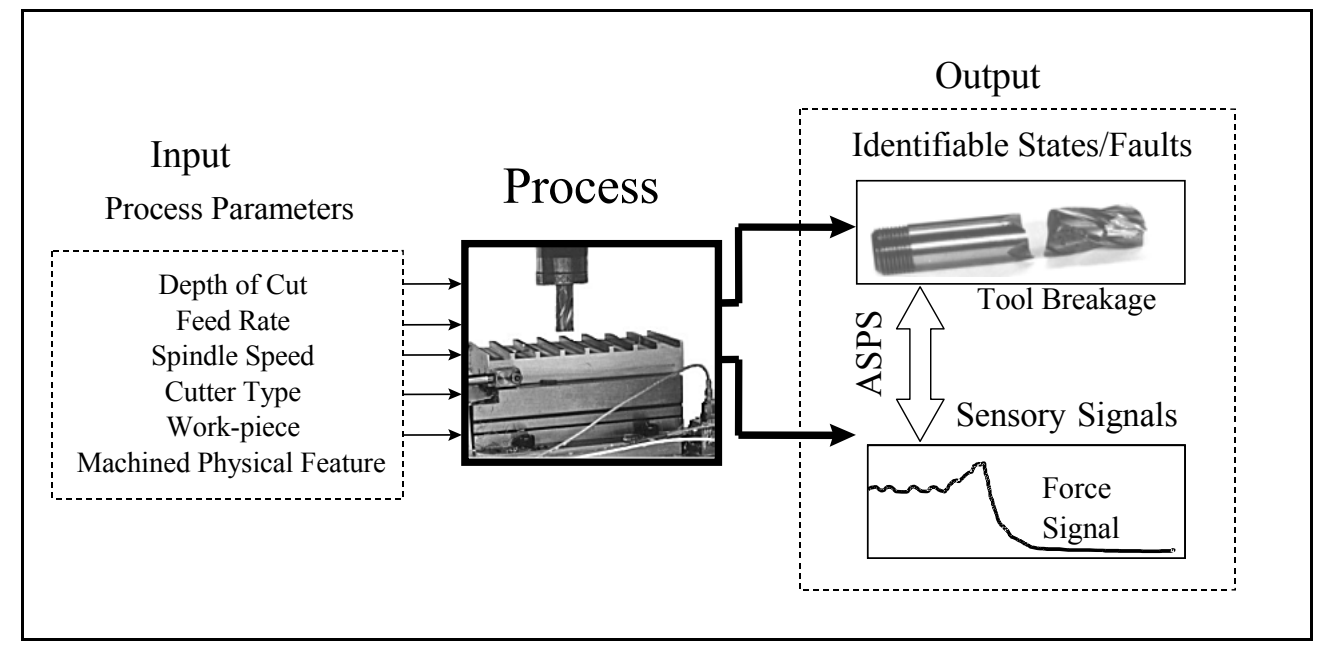

Figure 1: The "black box" concept and the way it can be applied for cutter breakage of end milling operation.

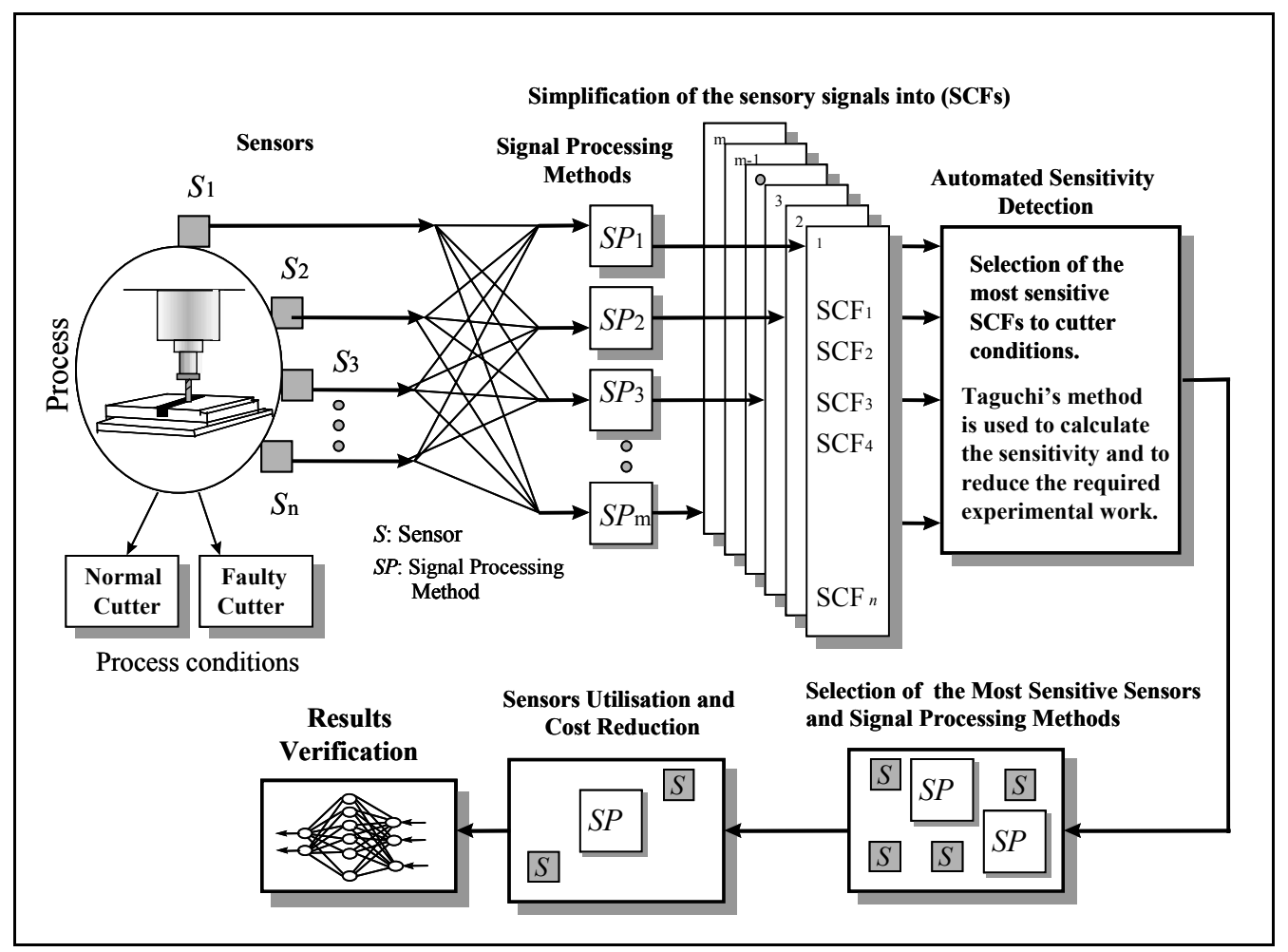

Figure 2: A simplified flow diagram of the ASPS approach using Taguchi's method. 


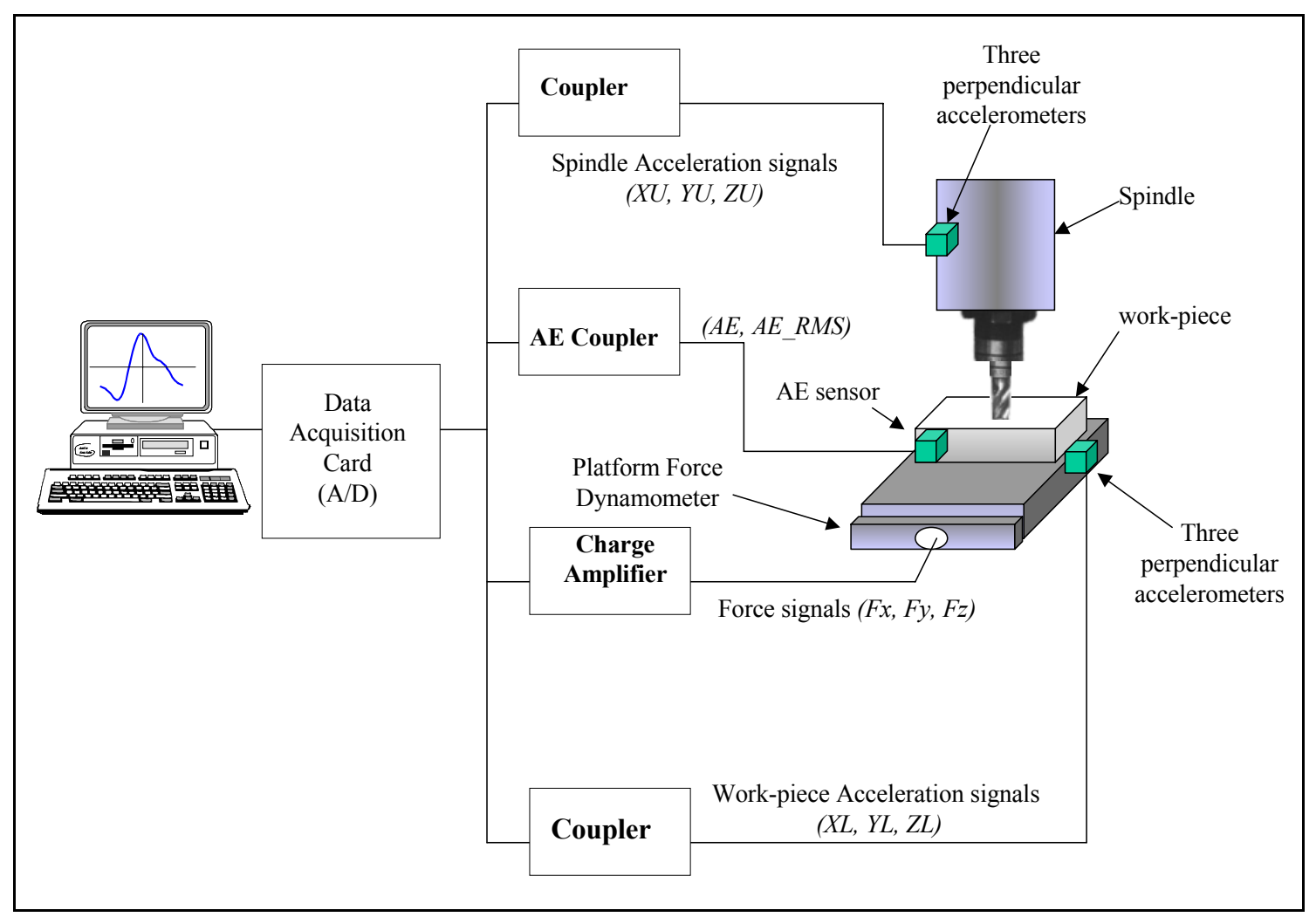

Figure 3: A schematic diagram of the experimental set-up of the data acquisition system. 


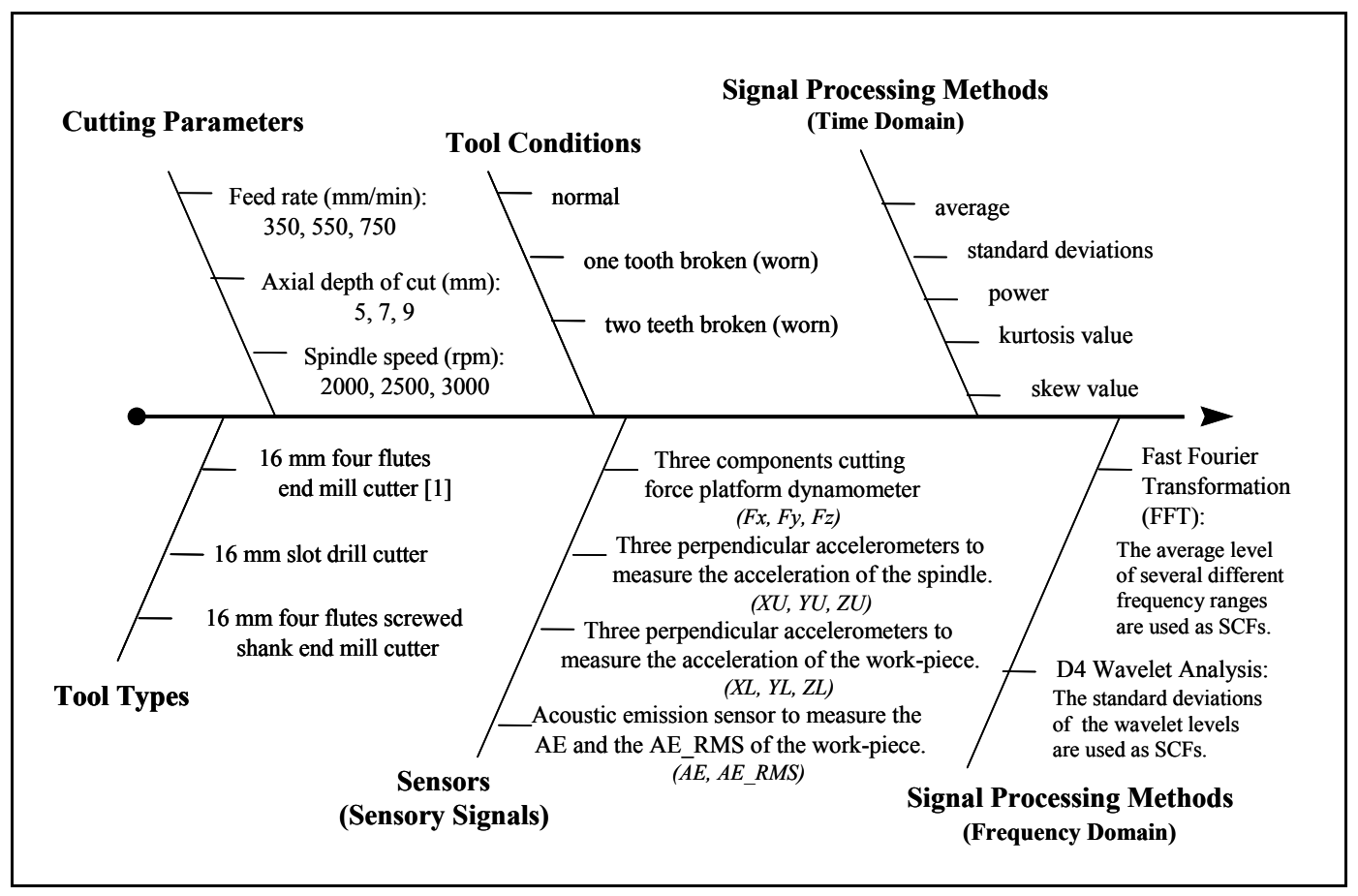

Figure 4: Summary of the complete experimental work. 


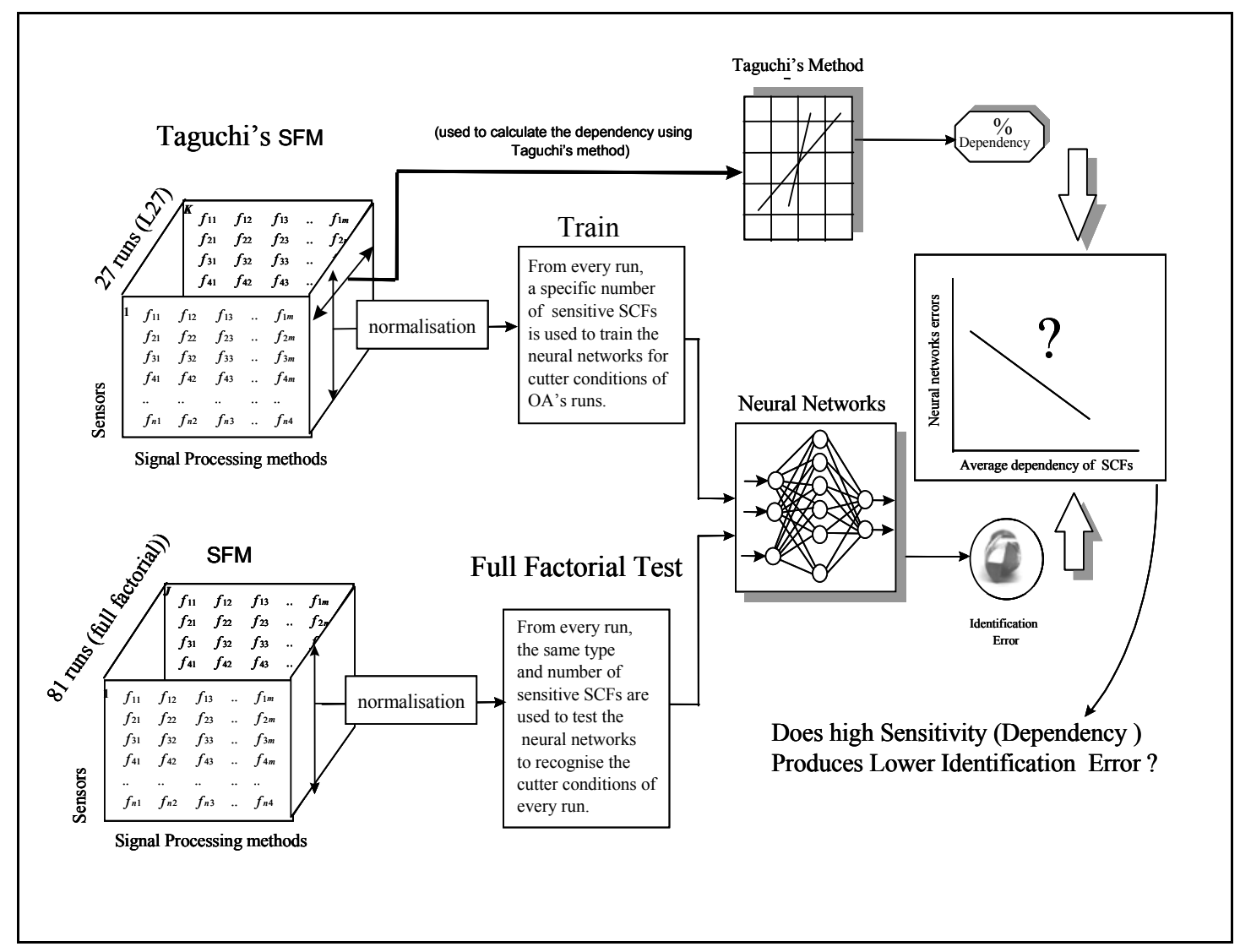

Figure 5: The relationship between the dependency values of the SCFs and the classification error of the neural networks can determine how successful the suggested approach is. 


\begin{tabular}{|l|l|l|}
\hline \multicolumn{1}{|c|}{ Neural networks } & \multicolumn{1}{c|}{ Type } & \multicolumn{1}{c|}{ Key Parameters } \\
\hline Back-propagation neural networks $(B P)$ & supervised & $\begin{array}{l}\text { learning rate }=0.001 ; \text { momentum } \\
=0.9 ; \text { target error }=0.01 ; \text { transfer } \\
\text { functions: sigmoid (hidden layer) } \\
\text { and linear (output layer). }\end{array}$ \\
\hline Radial Basis $(R B)$ & supervised & $\begin{array}{l}\text { target error }=0.01 ; \text { maximum } \\
\text { number of neurones }=1000 ; \text { spread } \\
\text { of radial basis function }=10 .\end{array}$ \\
\hline Competitive Neural Networks $(C N)$ & unsupervised & $\begin{array}{l}\text { learning rate }=0.1 ; \text { hidden layer size } \\
=50 ; \text { training iterations }=500 ; \text { bias } \\
\text { time constant }=0.99 .\end{array}$ \\
\hline Learning Vector Quantisation $(L V Q)$ & unsupervised & $\begin{array}{l}\text { learning rate }=0.05, \text { hidden layer } \\
\text { size }=50 ; \text { training iterations }=500 ; \\
\text { bias time constant }=0.99 .\end{array}$ \\
\hline
\end{tabular}

Figure 6: The four implemented neural networks.

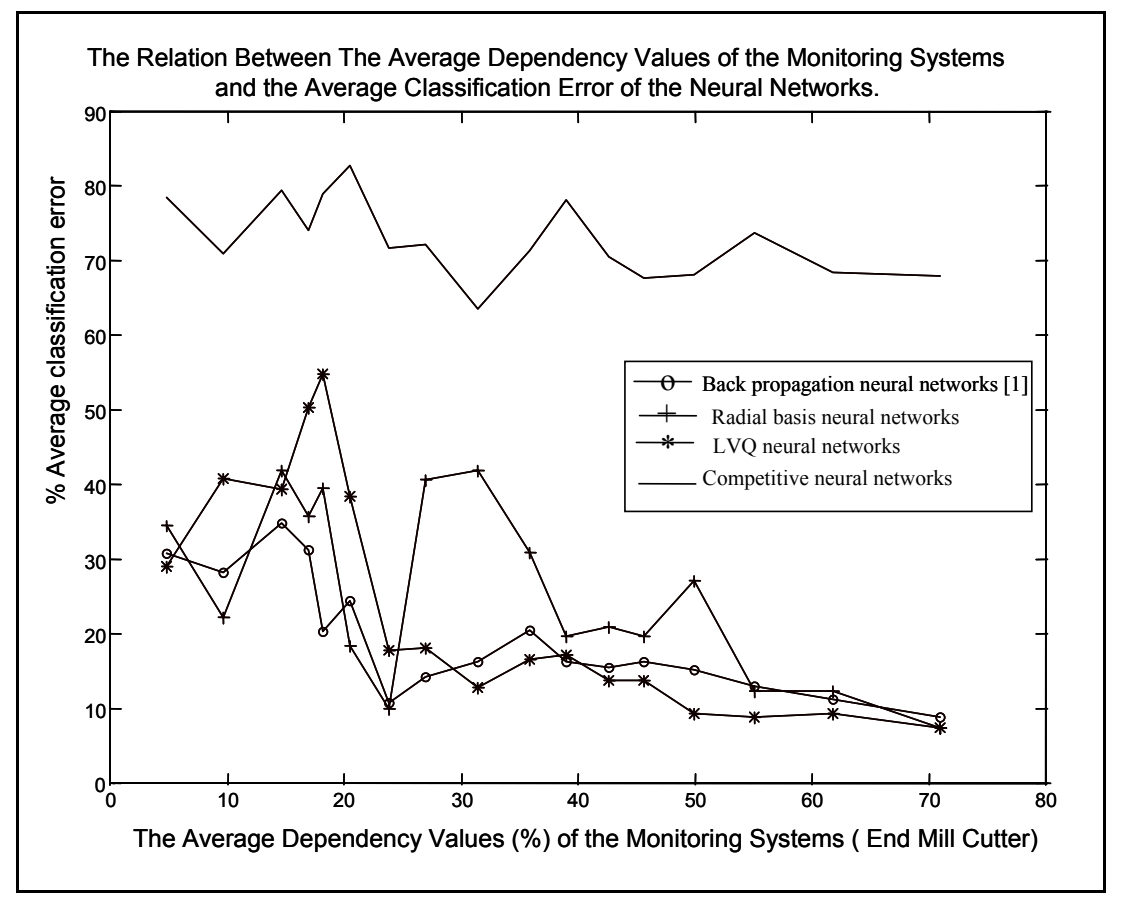

Figure 7: The classification error of neural networks for the end mill cutter. 


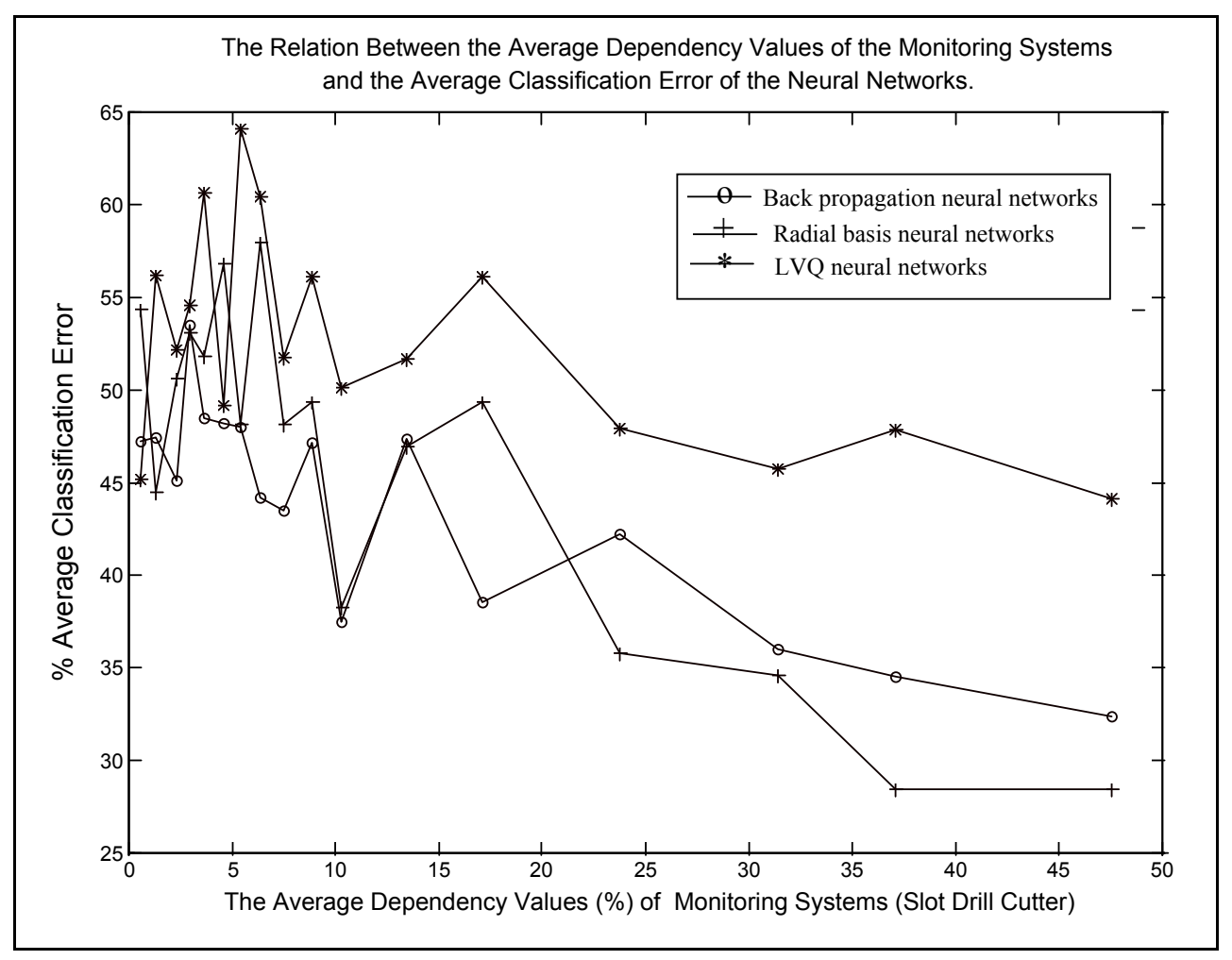

Figure 8: The classification error of neural networks for the Slot Drill cutter.

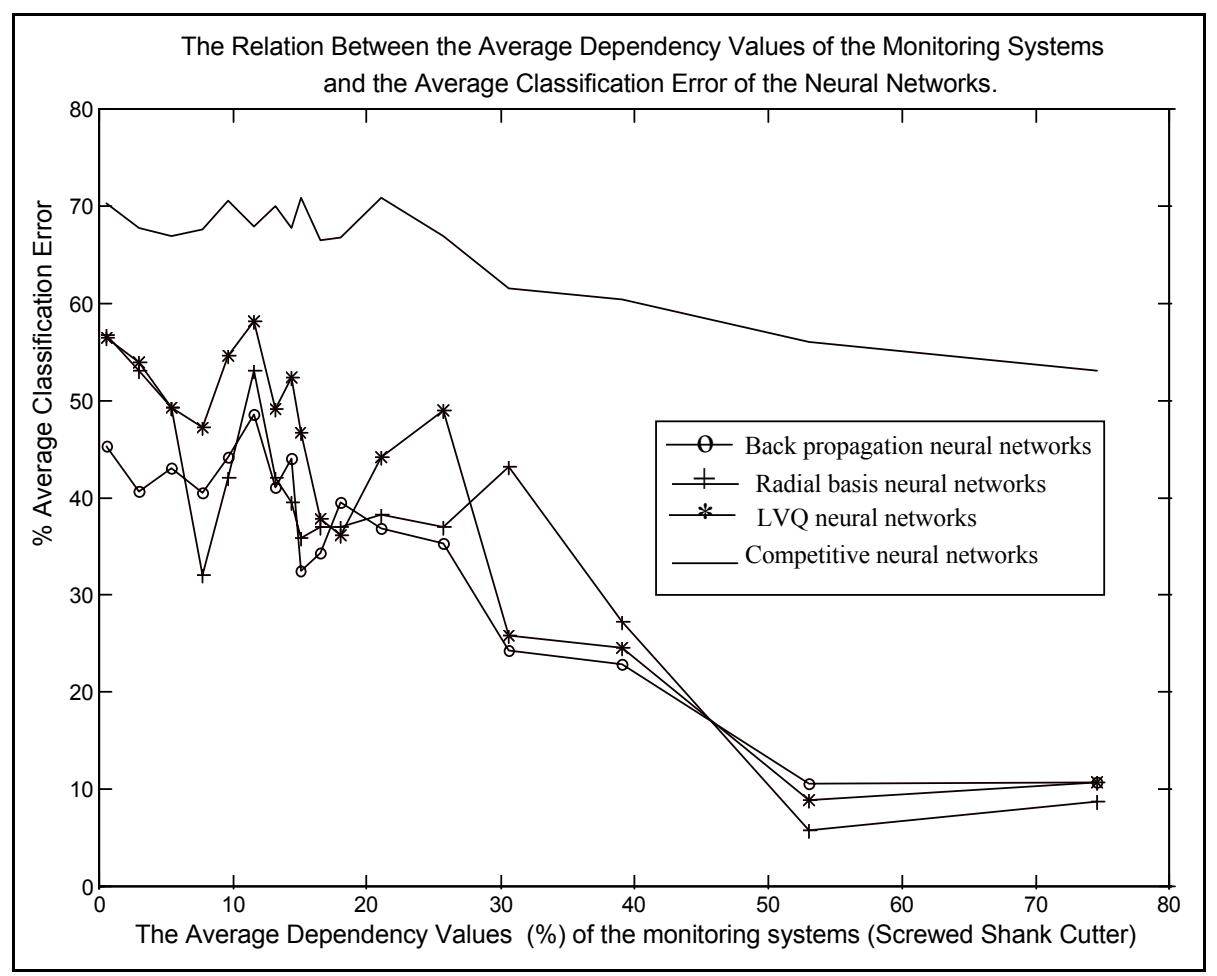

Figure 9: The classification error of neural networks for the screwed shank cutter. 


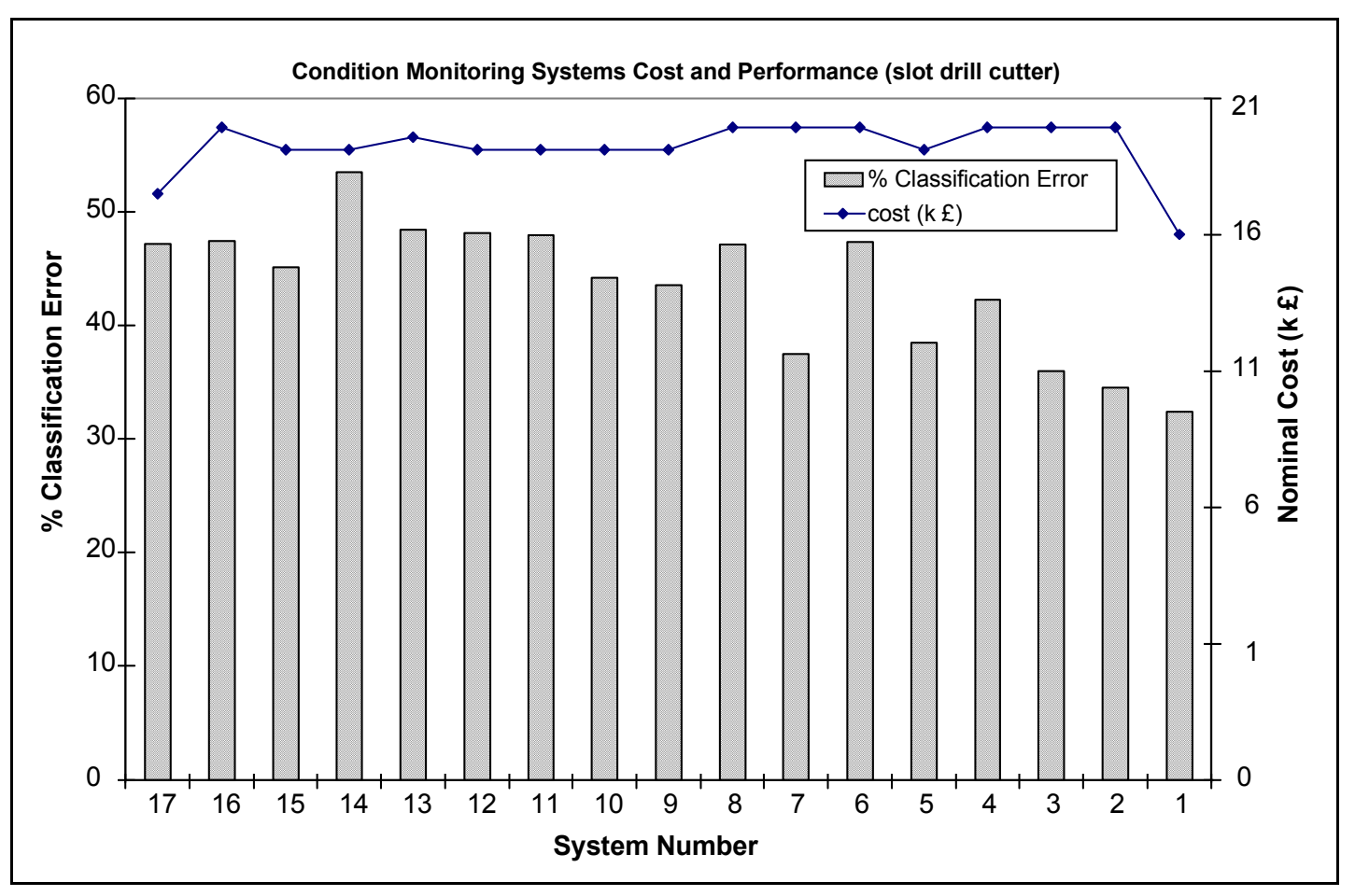

Figure 10: Comparison between the performance of the systems and the associated costs (slot drill cutter).

\begin{tabular}{|c|c|c|c|c|}
\hline \multirow[b]{2}{*}{ Monitoring Systems } & \multicolumn{2}{|c|}{ Slot Drill Cutter } & \multicolumn{2}{|c|}{ Screwed Shank Cutter } \\
\hline & System 1 & $\begin{array}{c}\text { Optimised } \\
\text { System } \\
\text { (from systems 1 } \\
\text { and 2) } \\
\end{array}$ & System 1 & $\begin{array}{l}\text { Optimised System (from } \\
\text { systems } 1 \text { and 2) }\end{array}$ \\
\hline Sensors & SU (\%) & SU (\%) & SU (\%) & SU (\%) \\
\hline Dynamometer & $10 \%$ & -- & $36.67 \%$ & $46.67 \%$ \\
\hline XL & -- & -- & -- & -- \\
\hline YL & -- & -- & $5 \%$ & -- \\
\hline ZL & -- & -- & -- & -- \\
\hline $\mathrm{XU}$ & -- & -- & $5 \%$ & -- \\
\hline YU & -- & -- & -- & \\
\hline $\mathrm{ZU}$ & -- & -- & $10 \%$ & -- \\
\hline $\mathrm{AE}$ & $80 \%$ & $100 \%$ & -- & -- \\
\hline $\begin{array}{c}\text { (SUA) } \\
\text { Overall Average Utilisation }\end{array}$ & $45 \%$ & $100 \%$ & $14.17 \%$ & $46.67 \%$ \\
\hline Average Dependency (\%) & $47.54 \%$ & $45.1 \%$ & $74.5 \%$ & $74 \%$ \\
\hline $\begin{array}{l}\text { Classification Error (\%) } \\
\end{array}$ & $32.5 \%$ & $37 \%$ & $11 \%$ & $11.5 \%$ \\
\hline Cost (f) & $£ 16,009$ & $£ 809$ & $£ 17,152$ & $£ 15,200$ \\
\hline
\end{tabular}

Figure 11: The utilisation of sensors (slot drill and screwed shank cutters) 


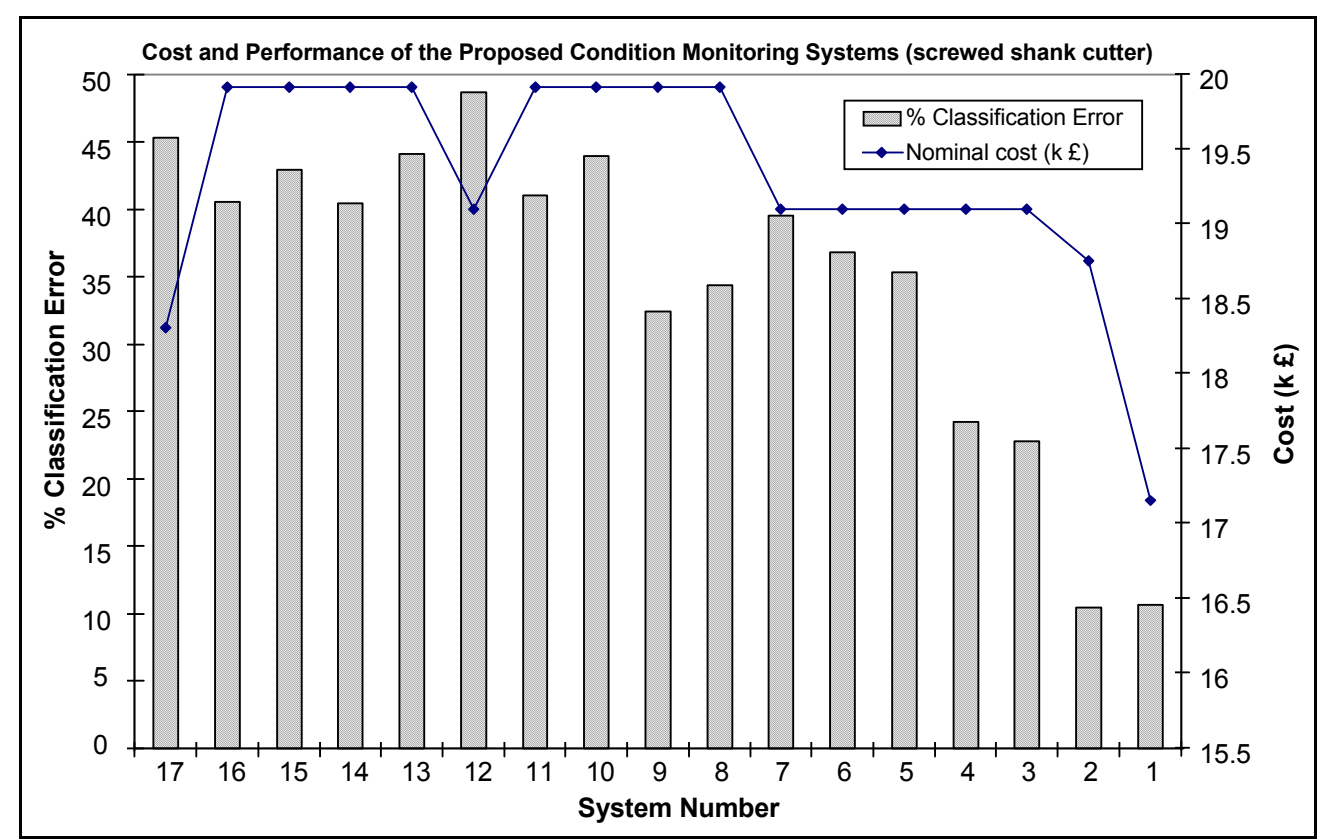

Figure 12: A comparison between the performance of the systems and their associated cost (screwed shank cutter). 